\title{
Estructuración de una unidad de mercadeo en una institución de educación superior en Barranquilla ${ }^{1}$ \\ Structuring of a marketing unit in institution of higher education (Barranquilla-Colombia)
}

DOI: http://dx.doi.org/10.17981/econcuc.40.1.2019.01

Artículo de investigación. Fecha de recepción: 28/09/2018 Fecha de aceptación: 26/12/2018

Eimys Vannesa Romero-Corro

Universidad Simón Bolívar. (Barranquilla, Colombia)

eimysr@gmail.com

Uliana Contreras-Buelvas

Universidad Simón Bolívar. (Barranquilla, Colombia)

ulianacontreras@hotmail.com

Para citar este artículo:

Romero-Corro, E. y Contreras-Buelvas, U. (2019). Estructuración de una unidad de mercadeo en una institución de educación superior en Barranquilla. Económicas CUC, 40(1). 9-26. http://doi.org/10.17981/econcuc.40.1.2019.01

\section{Resumen}

El desarrollo económico y social avanza a ritmos agigantados transformando con él las condiciones de vida de toda una nación, lo cual incluye a todos los sectores que componen un país, en este sentido, el sector educativo funge un rol muy importante ya que, hoy en día este sector no solo apunta hacia su razón de ser por naturaleza: educación e investigación sino que además apunta hacia otros objetivos en común como el crecimiento en el mercado, en este entendido, el presente artículo presenta una propuesta de diseño para una unidad de mercadeo debidamente estructurada en una institución de educación superior, encargada de coordinar y mejorar sus esfuerzos en marketing, basándose en sus objetivos organizacionales. El estudio fue de tipo descriptivo con método deductivo utilizado el paradigma cualitativo; permitiendo identificar las debilidades críticas del proceso de comunicación asociadas a la inexistencia de una unidad de Mercadeo; abarcando al personal administrativo de instituciones de educación superior encargados de los procesos ligados al mercadeo, y expertos en el área de mercadeo. A partir del análisis de la información primaria y secundaria recolectada se presentan los requisitos más importantes para plantear el diseño de la unidad de mercadeo con todos los procesos internos en materia de marketing y las funciones y estructura acorde a los requerimientos que tiene un instituto de educación como organización. Se concluye que las estrategias de marketing tradicional y las estrategias de promoción en medios digitales realizadas por la institución han arrojado resultados positivos en el transcurso del tiempo, sin embargo, deben ejecutarse con mayor coordinación.

Palabras claves: Estrategia; estructura organizacional; gestión de mercadeo; institución de educación superior; unidad de mercadeo.

\begin{abstract}
Economic and social development advances at gigantic rates, transforming with it the living conditions of an entire nation, which includes all the sectors that make up a country, in this sense, the educational sector plays a very important role since, today This sector not only points to its reason for being by nature: education and research, but also points to other common objectives such as growth in the market. In this understanding, this article presents a design proposal for a required marketing unit. Structured in a higher education institution, in charge of coordinating and improving your marketing efforts, controls your organizational objectives. The study was descriptive with a deductive method using the qualitative paradigm; We identified the critical weaknesses of the communication process associated with the absence of a Marketing unit; covering the administrative staff of higher education institutions in charge of the processes related to marketing, and experts in the area of marketing. From the analysis of the primary and secondary information collected, the most important requirements are presented to propose the design of the marketing unit with all the internal processes in marketing and the functions and structure according to the requirements of an educational institute. as an organization. It is concluded that the traditional marketing strategies and action strategies in digital media carried out by the institution have yielded positive results over time, however, they must be executed with greater coordination.
\end{abstract}

Keywords: Estrategy; organizational structure; marketing management; higher education institution; marketing unit.

\footnotetext{
${ }^{1}$ Artículo de investigación científica derivado del proyecto de investigación "propuesta de creación de la unidad de mercadeo para una Institución de Educación Superior de Barranquilla”.
} 


\section{INTRODUCCIÓN}

La rapidez del desarrollo económico y social transforma las condiciones de vida de un país, a un ritmo tal, que cada uno de los sectores, particularmente la educación, debe prever la adaptación de los individuos al mundo que lo rodea. Según Hernández, Chumaceiro y Reyes (2016) las universidades tradicionalmente cumplen sus funciones de formación e investigación. Sin embargo, cada año surgen nuevas instituciones educativas, orientadas a diferentes enfoques, sectores sociales y económicos que apuntan a otros objetivos en común: el crecimiento en el mercado atrayendo al mayor número de individuos con un aporte social y al desarrollo económico contribuyendo a la formación integral.

Así entonces, como expresan Hernández y Chumaceiro (2008), "el nuevo rol de las universidades es discutido internacionalmente como exigencia de una sociedad con alto valor educativo y, en consecuencia, con mayores expectativas desde su entorno" (p. 81). De esta manera la implementación del marketing educativo, le permite a las instituciones gestionar su desarrollo y participación en el mercado, y al mismo tiempo se convierte en una necesidad para poder destacarse ante la competencia resaltando la oferta institucional y académica. Este modo de gestión provee herramientas para realizar investigaciones sociales que generen el diseño de estrategias, además de incrementar el número de estudiantes potenciales, además de posibilitar soluciones de tipo administrativas.

Esta es la razón por la cual colegios, centros educativos y Universidades le apuntan a implementar unidades de mercadeo en el interior de sus instituciones, las cuales les permitan gestionar su quehacer administrativo y dado que:
... el departamento de marketing aporta una visión esencial a la dirección del centro, pues mira hacia fuera. Su objetivo es ofrecer lo que las personas demandan o, dicho de otro modo, adaptar su proyecto a las necesidades, gustos y preferencias de las familias (Llorente, 2017, p. 14).

La Institución de educación superior (IES) estudiada, cuenta con 45 años en el mercado, acreditación de alta calidad, una amplia oferta académica dada por 17 programas de pregrado en las áreas de administración y negocios, ciencias básicas y biomédicas, ciencias jurídicas y sociales, ciencias de la salud e ingenierías; alrededor de 45 programas de posgrados entre especializaciones, maestrías y doctorados, además de educación continuada en diferentes áreas de estudio. Con un número de 10.613 estudiantes para el primer periodo del 2017. Presenta un gran direccionamiento de sus estrategias de crecimiento, y posicionamiento en el mejoramiento continuo de procesos administrativos, metodologías académicas, certificaciones de calidad, desarrollo profesional de sus funcionarios, un mejoramiento de su infraestructura física, con la ampliación y diseño de nuevos productos educativos (nuevas carreras de pregrado y posgrado), entre otros aspectos internos que le han permitido destacarse entre las mejores universidades del departamento del Atlántico y destacarse a nivel nacional en el ámbito de la investigación.

Pese a lo anterior, muchos de estos avances de se ven limitados al conocimiento de los que hacen parte del alma mater. Así mismo, al momento de realizar estrategias o definir procesos, las personas a cargo tienden a confundir ventas con marketing, a raiz del errado pensamiento de que son una misma acción, y es por esto importante tener en cuenta que "la venta es una actividad comercial que supone el uso de argumentos válidos 
y éticos para transferir la propiedad de un bien o servicio de una persona o (empresa) a otra (cliente-comprador)" (Rojas, 2013, p. 13) y el "marketing es un proceso originado en el cliente, a través del estudio de sus necesidades, continuar con un conjunto de actividades empresariales para desarrollar bienes y servicios con el fin de satisfacer aquellas necesidades y general una demanda permanente" (Rojas, 2013, p. 22). Por lo tanto, la importancia del marketing se hace evidente en el desempeño de las organizaciones y su buena gestión implica una ventaja competitiva en relación y para la misma, en palabras de Farías (2014), el marketing juega un papel fundamental en las organizaciones, y debe ser visto como una inversión de la cual se obtiene la satisfacción de los usuarios y una mejora sustancial de la percepción de estos hacia la marca.

Por consiguiente, el presente artículo fue resultado de un proyecto investigativo donde se planteaba en primera instancia la necesidad desde el interior de la universidad objeto de estudio, la existencia de políticas de difusión masiva para dar conocimiento a la comunidad en general de los progresos alcanzados por la entidad y acercar los servicios de la institución a los actuales y potenciales estudiantes, además de hacer necesarias las acciones coordinadas de mercadeo para enfocar la institución al mercado.

Considerando lo señalado anteriormente, es viable y necesaria la implementación de una Unidad de Mercadeo encargada de mancomunar estrategias, acciones y procesos, los cuales actualmente se vienen manejando aisladamente. La carencia de esta unidad implica que no hallan políticas claras de marketing, ni lineamientos a seguir para posicionar la marca en el territorio nacional. Para esto se parte del siguiente cuestionamiento, dando lugar a los resultados del desarrollo del proyecto aquí presentados:
¿Cuál sería la estructura pertinente para la creación de una unidad de Mercadeo en la Universidad Simón Bolívar? lo cual lleva finalmente a establecer la estructuración de la unidad de mercadeo que se ajusta a las características e intereses de las IES.

\section{Fundamentación TeÓRICA}

En las sociedades actuales, la universidad debe trascender desde lo interno y autónomo hacia el exterior y abierta a realidades e influencias exógenas, Para ello, la universidad debe tener la capacidad de combinar cambios institucionales formales, y así lograr influir a su exterior. (Chumaceiro, Hernández y Chirinos, 2016).

De esta forma se presenta la definición de marketing que la American Marketing Association (AMA, 1960) establece como el proceso de planificar y ejecutar la concepción del producto, precio, promoción y distribución de ideas, bienes y servicios, para crear intercambios que satisfagan tanto objetivos individuales como de las organizaciones, la universidad, para el caso particular del presente estudio. Esto implica que el marketing tiene una gran amplitud en sus campos de aplicación, y no solo puede, sino que debe, emplearse no solo en empresas con actividad comercial, sino en cualquier organización con un proceso de intercambio.

Entre los diferentes tipos de marketing se encuentran el marketing orientado a servicios, en el caso que compete este proyecto. Martínez, Ruiz y Escrivá (2014), afirman que esta orientación del marketing existe debido a las características diferenciales de los servicios respecto a los bienes y la forma en que estos son percibidos por los usuarios, haciendo que el marketing de productos no pueda aplicarse de igual manera a los servicios. Para llegar a la efectividad en la implementación de este tipo de marketing, se tienen en cuenta las siguientes estrategias (Tabla 1): 
TABLA 1.

Estrategias del marketing de servicios.

\begin{tabular}{cl}
\hline Hacer tangible el servicio & $\begin{array}{l}\text { Se trata de conseguir que el servicio sea captable por los sentidos creando } \\
\text { una representación física del mismo. Por ejemplo, ofreciendo carpetas o } \\
\text { bolígrafos con el logotipo de la empresa. }\end{array}$ \\
Identificar el servicio & $\begin{array}{l}\text { Hay que asociar el servicio a una imagen que permita diferenciarlo de } \\
\text { la competencia. Por ejemplo, mediante el uso de un determinado uniforme } \\
\text { por parte del personal o de una melodía que se asocie al servicio. }\end{array}$ \\
Diferenciar por calidad & $\begin{array}{l}\text { Consiste en ofrecer un valor añadido al servicio para que se diferencie de } \\
\text { la competencia por el nivel de calidad ofrecido. }\end{array}$ \\
Estandarizar el servicio & $\begin{array}{l}\text { Se trata de que el servicio ofrecido sea el mismo para todos los clientes y } \\
\text { se garantice un nivel estándar de calidad. }\end{array}$ \\
\hline
\end{tabular}

Fuente: elaboración propia.

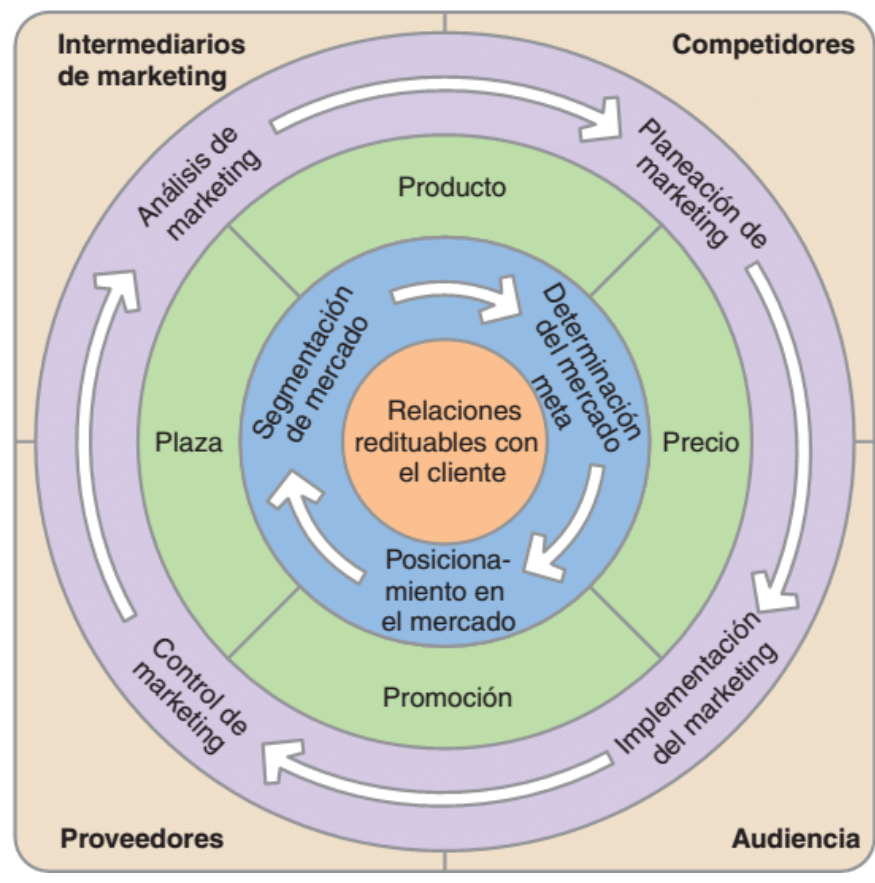

Figura 1. Proceso de la estrategia marketing. Fuente: Kotler y Armstrong (2008),

Por otro lado, Kotler y Armstrong (2008) proponen un modelo de proceso de marketing éxitoso para el competitivo mercado actual, conquistar clientes de la competencia, conservarlos y desarrollarlos mediante una entrega de mayor valor. Pero antes de poder satisfacer a estos usuarios, se debe entender primero sus necesidades y expectativas. Por lo tanto, el buen marketing requiere de un cuidadoso análisis del cliente.
Adicionalmente, la organización debe tener un equipo de personas a cargo que puedan desarrollar e implementar los procesos anteriormente planteados, y estos conformaran el departamento de marketing, que en palabras de Martinez, Ruiz y Escrivá, (2014), se encarga de estudiar el mercado en profundidad para elaborar la oferta que cubra sus necesidades y dársela a conocer a los clientes. 


\section{GERENTE DE MARKETING}

Figura 2. Estructura del departamento de marketing. Fuente: propuesta por Rojas (2013)

Asimismo, para Rojas (2013), una empresa, independientemente de su tamaño, debe reunir 3 factores que van a interactuar en su desempeño: objetivos, estructura y personas. En palabras de este mismo autor: "la organización es un proceso mediante el cual se asignan responsabilidades a las personas en diversos niveles de gestión (estructura) para que con la debida autoridad puedan ejecutar el plan de marketing y alcancen sus objetivos pre-establecidos" (p. ). Por consiguiente, nos propone una estructura de un departamento o unidad de mercadeo para estos casos (Figura 2).

De este mismo modo, es necesario tener en cuenta que el marketing moderno se organiza y se orienta en función de las necesidades de los clientes y de la empresa.

Rojas (2013) describe un panorama de las posibles funciones que puedan tener las personas a cargo de un departamento de mercadeo, como lo son:

- Definir un plan de acción.

- El gerente de mercadeo debe elegir el personal idóneo para la realización de cada actividad.

- Establecer la plaza o territorio donde va a actuar la fuerza de ventas, y este factor está relacionado directamente con la investigación previamente realizada, el presupuesto y el tiempo disponible.

- Es importante también definir el tiempo en el que se van a desarrollar las acciones del plan.

- Por último, y no menos importante, la provisión de un presupuesto adecuado para el desarrollo de los planes de marketing.

Por otro lado, y guardando cierta congruencia, Martínez, Ruiz y Escrivá (2014) proponen las siguientes posibles funciones del departamento de mercadeo:

- Analizar el mercado con el fin de conocer los puntos fuertes y débiles de la organización y detectar oportunidades y amenazas del entorno. También busca conocer las características y necesidades de los consumidores.

- Establecer los objetivos que desea conseguir, y diseñar las estrategias de marketing mix para alcanzarlos.

- Organizar los medios humanos y materiales para la puesta en práctica de las estrategias.

- Ejecutar las acciones previstas.

- Controlar el desarrollo del programa comercial.

$\mathrm{Y}$ partiendo de estas funciones, estos mismos autores, definen una organización para este tipo de departamento: 


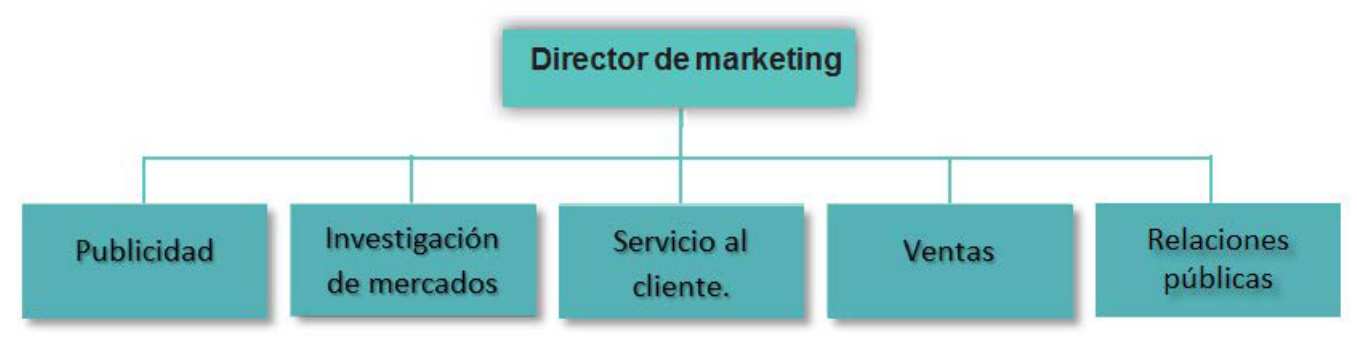

Figura 3. Organización del departamento de marketing.

Fuente: Martínez, Ruiz y Escrivá (2014)

Inclusive es pertinente que para el buen funcionamiento de una Unidad de mercadeo, debe existir un manual de funciones, el cual se define como "un libro que contiene lo más sustancial de un tema, y en este sentido, los Manuales son vitales para incrementar y aprovechar el cúmulo de conocimientos y experiencias de personas y organizaciones" (Álvarez 1996). Además, los manuales representan un medio de comunicación de las decisiones de la administración, concerniente a objetivos, funciones, relaciones, políticas, procedimientos, etc. En la actualidad, el volumen y la frecuencia de dichas decisiones continúa incrementándose en todas las instituciones, y con ello, los manuales como mediadores de comunicación cobran mayor relevancia (Rodríguez, 2012)

Por último y no menos importante, cabe recalcar que los factores a tener en cuenta para organizar o definir la estructura de una unidad o departamento de mercadeo con sus funciones y responsabilidades van a depender del tamaño de la empresa, del número de productos $\mathrm{y}$ marcas que se ofrezcan y de su nivel de complejidad, y de los mercados a los que se dirija, así como del sector en el que se trabaje.

\section{MetodoloGía}

El estudio que permitió el análisis de las herramientas necesarias para la elaboración del proyecto se desarrolló en el periodo comprendido entre febrero a agosto del año 2017, para lo cual se implementó una metodología descriptiva cualitativa.

El tipo de estudio para este proyecto fue descriptivo (Díaz, 2009) al situarlo sobre una base de conocimientos más sólida que los exploratorios. En este caso el problema ha alcanzado cierto nivel de claridad, pero aún se necesita información para poder llegar a establecer caminos que conduzcan al esclarecimiento de relaciones causales. Se emplea el método deductivo (Bernal, 2006), que permite tomar conclusiones generales del estudio para obtener explicaciones y desarrollar herramientas particulares.

Esta investigación tuvo un paradigma cualitativo, teniendo en cuenta la definición que plantea Denzin y Lincon (1994)donde se integran diferentes aspectos que la hacen multi-paradigmática en su enfoque. Es practicada por la sensibilidad al valor del enfoque multimetódico y esta sometida a la perspectiva naturalista y a la comprensión interpretativa de la experiencia humana.

Para el desarrollo de la investigación se utilizaron e implementaron las siguientes fuentes y técnicas de recolección de información (Tabla 2). 
TABLA 2

Fuentes y técnicas de recolección de información

\begin{tabular}{ll}
\hline & - Observación directa \\
Fuentes & Entrevistas a \\
Primarias & Profundidad \\
& Técnica Delphi \\
& Fuentes secundarias \\
& internas: \\
& Estructura \\
& organizacional. \\
& Análisis de contenido \\
& institucional. \\
Fuentes & Fuentes secundarias \\
externas: & Documentos científicos \\
& Investigaciones \\
& realizadas \\
& Teorías de autores \\
& reconocidos \\
\hline
\end{tabular}

Fuente: Elaboración del autor.

\section{Determinación de la Muestra}

El tamaño de la muestra se seleccionó a través de un muestreo no probabilístico específico para las técnicas a implementar (Tabla 3).

TABLA 3

Determinación de la muestra

\begin{tabular}{ll}
\hline & $\begin{array}{l}\text { Muestreo no probabilístico } \\
\text { a conveniencia (Malhotra, } \\
\text { 2004) donde los } \\
\text { entrevistados son personas } \\
\text { Entrevistas a }\end{array}$ \\
en variedad de cargos en \\
las diferentes áreas de la \\
institución y con cierta \\
influencia en el tema de \\
investigación. \\
Realizada por 3 expertos \\
seleccionados a través de un \\
muestreo no probabilístico \\
a conveniencia, teniendo \\
en cuenta su conocimiento \\
y trayectoria en temas \\
procesos y estrategias de \\
mercadeo.
\end{tabular}

Fuente: Elaboración del autor.

\section{Procesamiento de la Información}

El trabajo de campo de la investigación comprendió como primer paso, la realización de entrevistas al personal encargado de realizar actividades de marketing o afines en la institución objeto de estudio, con el fin de conocer más a fondo los procesos de mercadeo que actualmente se realizan en esta. De igual forma, se realizaron pruebas tipo método Delphi a expertos en el tema de estudio, en función de conocer la opinión de estos acerca de la importancia o necesidad de la implementación de una unidad de mercadeo en una institución de educación superior, para corroborar la pertinencia de esta en el proyecto plateado, y así mismo, establecer bases a partir de la experiencia para el diseño de la estructura y funciones que debe cumplir la unidad de mercadeo propuesta.

Para el procesamiento de los datos recopilados se desarrollaron los siguientes pasos:

\section{1) Codificación de los datos recopilados en el trabajo de campo}

Como primer paso los datos recopilados con los diferentes instrumentos se organizaron y sistematizaron con la finalidad de contar con el conjunto de datos pertinentes para el proceso de análisis.

\section{2) Resumen de la información cualitativa}

A partir del producto de las entrevistas y la técnica Delphi por expertos aplicadas al personal de la empresa, se elaboró un resumen de cada técnica sintetizando los principales aspectos discutidos en relación al proceso investigativo, con el fin de establecer la perspectiva de cada uno frente a las temáticas de los procesos de mercadeo dentro de las instituciones de educación superior. 


\section{3) Procesamiento en software especializado.}

Para el caso del resumen de las entrevistas aplicadas el procesamiento se emplea el software NVIVO. V.10 para Windows.

\section{4) Definición de criterios para interpretación de resultados.}

Para el análisis e interpretación de la información derivada del proceso de recopilación de la información del trabajo de campo se ha tenido en cuenta: la pertinencia de la teoría referente a la temática de procesos de mercadeo, estructura organizacional de unidades de mercadeo y el manual de funciones en sintonía al contexto donde se encuentra inmersa la empresa.

\section{Resultados}

En los anexos de este artículo se presenta la información textual extraída de las técnicas de recolección aplicadas sintetizadas detalladamente. A continuación se exponen los aportes más importantes de cada uno, que posteriormente contribuyen a establecer la estructura, modelo y requerimientos necesarios para la propuesta en mención.

\section{Análisis de los resultados}

Esta información extraída de las técnicas de investigación fue útil, en principal medida, para contrarrestar y reafirmar la concepción inicial del proyecto. Así mismo, permite el análisis a profundidad del modelo de trabajo empleado por la institución en el periodo de realización de la investigación, por lo que fue posible estudiar y entender los procesos realizados al interior de la IES para definir y ejecutar las acciones de mercadeo y de esta manera, identificar las fortalezas y falencias en dicha área, que se presentan más adelante mediante una matriz FODA; de esta manera es posible identificar las características necesarias para el diseño de la estructura de la unidad de mercadeo acorde a las particularidades de la institución.

Por otro lado, mediante el método Delphi, se pretende establecer una guía para la ejecución de estrategias de mercadeo de forma óptima, bajo estructuración de departamentos y/o unidades de mercadeo, así como respaldar por voces expertas la pertinencia y la estructura adecuada para este tipo de propuestas ejecutadas en IES. De esta manera la opinión de estos expertos y los apuntes dados por su experiencia profesional, respaldan este proyecto y sustentan su aplicabilidad permitiendo encontrar las herramientas indispensables requeridas para una unidad de mercadeo.

En este sentido, a partir de la interpretación de la información recopilada, se presenta en primera instancia, el análisis FODA, que confrontando lo afirmado por Ponce (2006), permite evaluar los factores fuertes y débiles en conjunto, diagnosticando la situación interna de las IES como organización, así como su evaluación externa, es decir, las oportunidades y amenazas. También es posible obtener una perspectiva general de la situación estratégica de la institución.

\section{Análisis FODA orientado al Marketing de la IES Objeto de Estudio}

\section{1) Fortalezas}

- Profesionales en comunicación social, diseño gráfico y administración de empresas que direccionan las estrategias.

- Recursos económicos para realizar inversión en marketing.

- Espacios publicitarios en los diarios regionales.

- Cuñas radiales en emisoras locales.

- Potencial de estudiantes para marketing de boca a boca.

- Diseño y producción de material POP.

- Precios económicos por una calidad excelente en la educación. 


\section{2) Debilidades}

- Carencia de la Unidad de mercadeo.

- Falta de innovación en las estrategias de promoción.

- No se realizan investigaciones de mercados.

- No existe una política de incentivos de becas.

- No se promueve el sentido de pertenencia de los funcionarios, docentes y estudiantes.

- No cuenta con profesionales en Ingeniería de mercados (carrera ofertada por la misma institución).

\section{3) Oportunidades}

- Penetrar nuevos mercados fuera de la región Caribe.

- Posicionar la marca en la región Caribe.

- Aumentar el número de estudiantes que ingresan en el semestre.

- Ser líder en el nicho me mercado al cual se dirigen.

\section{4)Amenazas}

- Reducir el número de estudiantes que ingresan por semestre.

- Eliminación de programas de pregrado o posgrado por falta de reconocimiento.

La institución objeto de estudio presenta amplias competencias en materia de mercadeo, que sin duda son sus fortalezas más destacables. Éstas le permiten desarrollar estrategias efectivas teniendo suficientes recursos disponibles, tanto humanos como económicos y, por supuesto, enlaces de comunicación para la comunicación de su oferta y sus estrategias. No obstante, presenta algunas falencias referentes a la gestión y organización interna para la ejecución de sus estrategias, ocasionando que no se desarrollen a cabalidad y no se noten los esfuerzos integrados de forma efectiva.
$\mathrm{Al}$ no existir un departamento integrado de mercadeo, los esfuerzos en muchas ocasiones se desvían y pierden el foco común además de generar desequilibrio y una falta de proyección, desatendiendo aspectos importantes como el estudio permanente del mercado y la innovación en las estrategias de marketing.

Adicionalmente, es importante destacar como a pesar del entorno competitivo al que se encuentra enfrentada la IES, dadas las fortalezas, capacidades internas y otros factores del entorno, se genera un ámbito favorable que permite destacar en el mercado educativo; y sin dudas, la opción de posicionarse en él, siempre y cuando sea capaz de gestionar efectivamente las variables internas y externas que se le presenten.

Por otra parte, para respaldar la pertinencia del área de mercadeo, se presenta un breve contraste en relación al anterior DOFA con otras instituciones de educación superior donde compiten en el mismo mercado estudiado, permitiendo determinar los esfuerzos de cada una de éstas en el desarrollo de estrategias e implementación de herramientas para la gestión del mercado con su posicionamiento y participación, considerando que las empresas exitosas además de descifrar a sus consumidores o clientes, son capaces de aprender de la competencia (Jiménez, 2012). Por esta razón, pertinentemente se toma como referencia lo siguiente:

- En la ciudad de Barranquilla existen ocho (8) universidades, incluyendo la universidad objeto de estudio:

- Cabe destacar que la líder del mercado es la Institución A, por los importantes logros a nivel académico, las certificaciones obtenidas, los premios de los docentes y la excelente gestión que hacen en los medios masivos y alternativos de comunicación para posicionar su marca institucional. 
Algunas de las actividades que realizan:

- Promoción masiva de sus programas de becas.

- Divulgación de las historias exitosas de sus estudiantes para atraer a futuros aspirantes.

- Material publicitario en eventos de cubrimiento televisivo.

- Las instalaciones son sedes de importantes eventos de moda.

- Avisos publicitarios en los paraderos de buses y en vallas estratégicas de la ciudad.

- Excelente manejo del material POP.

Otra universidad que realiza gestiones importantes de mercadeo es la Institución B., la cual desde el Departamento de Comunicaciones de la misma dirige la coordinación de publicidad y desarrolla estrategias de posicionamiento más cercanas a sus potenciales clientes como son:

- Anuncios publicitarios en periódicos y canales de televisión municipales.

- Participación en eventos de cubrimiento televisivo.

- Patrocinio de eventos públicos.

- Difusión de todos sus logros en el periódico regional.

- Página web interactiva y rápida.

- Excelente manejo de redes sociales.

\section{Presentación de resultados por objetivos}

Ahora bien, se presentan los resultados finales del proyecto de investigación sintetizados en el desarrollo de los objetivos planteados para la estructuración de la unidad de mercadeo:

\section{1) Procesos asociados a la gestión de mercadeo de la Institución de Educación Superior.}

La necesidad de determinar los requerimientos del cliente y satisfacerlos se ha convertido en el principal objetivo de las empresas, esto claramente se puede lograr de diversas formas. Sin embargo, Kotler y Armstrong (2008) a través de su modelo de proceso de marketing plantean acciones conjuntas que permiten tener éxito en el competitivo mercado actual, integradas al departamento de marketing, el cual se encarga de estudiar el mercado en profundidad para elaborar la oferta que cubra sus necesidades y ponerla en conocimiento de la empresa (Martinez, Ruiz y Escrivá, 2014). Gestionar estas actividades, relacionadas con el departamento de marketing, ha llegado a ser el fin de cualquier sistema de calidad y, por ende, del resto de departamentos de la organización.

En función de lo anterior, a continuación se presentan las acciones implementadas dentro de la institución para el desarrollo de sus procesos de marketing, donde se recalca que las acciones se realizan por diferentes departamentos trabajando de forma independiente, independiente al trabajo en forma paralela. Sin embargo, se hace evidente la necesidad de unificar ciertas acciones para obtener resultados óptimos enfocados a los siguientes objetivos en común:

- Departamentos que de forma aislada gestionan las acciones de marketing desarrolladas por la institución:

1. Departamento de Comunicación Organizacional: División donde se encentran profesionales del diseño gráfico encargados de hacer las piezas publicitarias, y comunicadores social en función de sistematizar la información de la organización y hacer las publicaciones de las noticias en la página web y redes sociales.

2. Historia Gráfica: En esta división se encargan de recopilar todas las imágenes de los eventos de la universidad para luego ser publicadas en libros o redes sociales. 
3. Departamento de Comunicación y Divulgación: Este departamento registra en video las actividades de la institución estudiada. Son los encargados de enviar los anuncios a los diarios y emisoras.

4. Departamento de Promoción y Admisiones: se encarga de realizar actividades en los colegios y participar en ferias para atraer estudiantes, además se encarga del proceso de admisión y de asignación de becas para el primer semestre.

5. Departamento de Sindicatura: Sin ser profesionales afines, en este departamento es donde se toman importantes decisiones sobre marketing; como el tipo de piezas a imprimir, el material POP (Punto de Venta) a utilizar, y en ocasiones hasta intervienen en el diseño de plegables y volantes institucionales.

- En cuanto a los recursos publicitarios, la Universidad objeto de estudio cuenta con profesionales en diseño gráfico encargados de realizar las piezas institucionales, además de poseer herramientas y contactos para desarrollar sus acciones publicitarias:

1. Página web interactiva.

2. Plegables con información de programas y servicios.

3. Anuncios en periódicos y emisoras.

4. Material POP.

5. Programa de televisión.

6. Presencia en medios digitales.

- Es evidente que la IES realiza estrategias o acciones descoordinadas sin un alto impacto en el mercado; estrategias bastante generales; información obtenida a partir de las fuentes investigadas, porque institucionalmente se se ha asumido y definido que el grupo objetivo está dado por estudiantes de estratos de 1 al 3, sin ahondar en una segmentación de mercados más estructurada, limitando la información y las herramientas para acciones de marketing más efectivas, por tanto, es claro la carencia de una buena segmentación que le permita organizar efectivamente el público objetivo, teniendo en cuenta como este puede generar subsegmentaciones más amplias.

- Por último, en materia de satisfacción, fidelización y captación de clientes, ante la situación actual de los mercados, "adquirir nuevos clientes es cada vez más costoso y peligroso" Schnarch (2013, p. 120) y resulta mejor fidelizar a los clientes actuales que captarlos. Analizando esto, las estrategias de fidelización de estudiantes se quedan cortas en temas netamente académicos y no siempre generan buenas perspectivas por parte de estos, haciendo posible reforzarlo con acciones contundentes y específicas de servicio al cliente por estar actualmente bastante desatendido este tema en la institución. Además esto generaría una mayor facilidad para estrategias como el voz a voz.

\section{2) Funciones generales que debe asumir} la unidad de mercadeo de la IES.

Teniendo en cuenta estos puntos específicos de los referentes bibliográficos, como las funciones del departamento de mercadeo expuestas por Martinez, Ruiz y Escrivá (2014). Así como los resultados de las entrevistas al personal experto en mercadeo y las necesidades de la IES se lograron determinar las siguientes funciones generales que deberá asumir la Unidad de Mercadeo de dicha institución, en caso de ser implementada la presente propuesta: 
TABLA 4

Funciones generales de la Unidad de mercadeo

Funciones generales de la unidad de mercadeo de la IES

1. Desarrollar actividades de investigación de mercados, que incluyan análisis y segmentación de mercado, determinación del mercado meta, que arrojaran estrategias de posicionamiento en el mercado.

2. Planificar reuniones de trabajo con personal administrativo, estudiantes y profesores, por facultad; para revisar y actualizar procedimientos, planes de estudio y demás, con respecto a los resultados de investigaciones realizadas.

3. Revisión y reestructuración de costos en inscripciones, matriculas, créditos, certificados, cursos de énfasis, etc.; a efecto de coordinar con la vicerrectoría académica.

Acciones conjuntas con el departamento de admisiones.

Acciones conjuntas con los representantes académicos de la institución.

Coordinación con sala general y vicerrectoría académica y financiera.

4. Coordinar las actividades de comunicación externa (promoción, publicidad, relaciones públicas) para optimizar los recursos y dar a conocer de una manera efectiva la oferta académica de la institución, y además los procesos, investigaciones, reconocimientos, etc., de la misma.

5. Coordinar las actividades de comunicación interna de la institución.

6. Establecer la plaza o territorio donde va a actuar la fuerza admisiones (captación y registro de estudiantes), y este factor está relacionado directamente con la investigación previamente realizada, el presupuesto y el tiempo disponible.

Acciones conjuntas con el departamento de admisiones.

7. Analizar, planear, implementar y controlar las estrategias dirigidas a crear, formar y mantener relaciones óptimas con usuarios (estudiantes).

8. Realizar constantemente estrategias de benchmarking que permitan evaluar los productos, servicios y procesos de trabajo de instituciones reconocidas, y la vez conocer aquellos competidores más duros.

9. Provisión de un timing y presupuesto adecuado para el desarrollo de los planes y procesos de mercadeo a realizar.

Acciones conjuntas vicerrectoría financiera, admisiones y matriculas.

10. Revisión y control del desarrollo y resultado de cada plan de acción de mercadeo.

Fuente: Elaboración del autor

\section{3) Estructura organizacional de la unidad de mercadeo de la IES.}

A partir de la investigación realizada, el análisis de las necesidades específicas de la IES estudiada y la estructura del departamento de marketing expuesta por Rojas (2013). Se presenta la estructura organizacional y el manual de funciones para la unidad de mercadeo propuesta. 


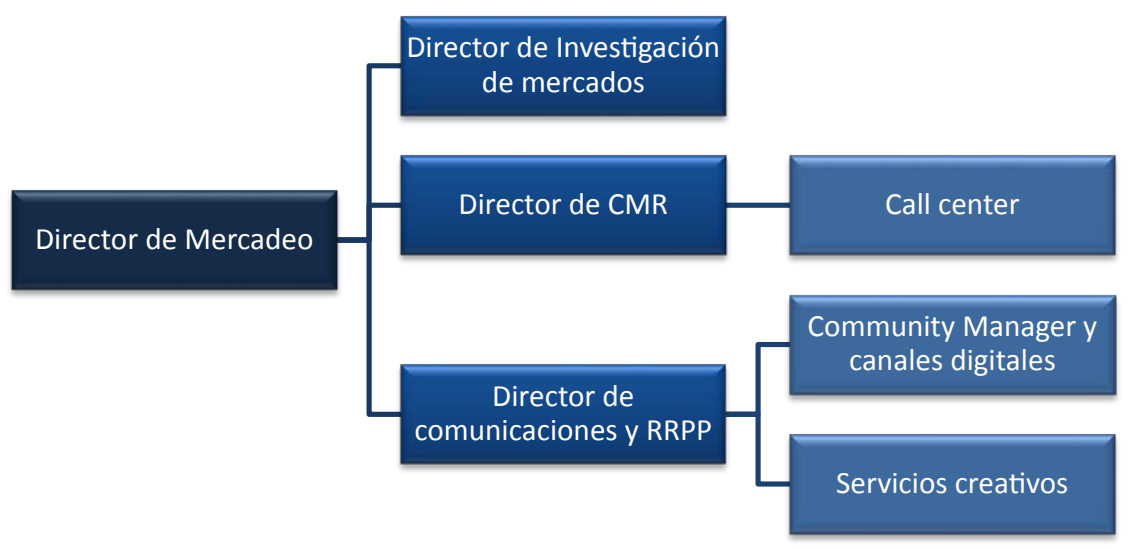

Fig. 4. Estructura organizacional para unidad de mercadeo.

Fuente: Elaboración del autor.

Manual especifico DE FUnCIONES DE LA UnidAD

DE MERCAdEO DE LA Universidad Simón Bolívar

\begin{tabular}{|c|c|}
\hline \multicolumn{2}{|c|}{$\begin{array}{l}\text { MANUAL ESPECIFICO DE FUNCIONES DE LA UNIDAD DE MERCADEO } \\
\text { DE LA UNIVERSIDAD SIMÓN BOLÍVAR }\end{array}$} \\
\hline \multicolumn{2}{|l|}{ I. IDENTIFICACIÓN DEL CARGO } \\
\hline Cargo: & Director de mercadeo \\
\hline Área: & Mercadeo \\
\hline \multicolumn{2}{|l|}{ II. OBJETIVOS } \\
\hline \multicolumn{2}{|c|}{$\begin{array}{l}\text { Velar por el logro de los objetivos institucionales direccionando las estrategias hacia la rentabilidad, } \\
\text { posicionamiento y sostenimiento en el mercado, a través de la integración de todas los cargos de la Unidad y el } \\
\text { mantenimiento de un clima y cultura motivadora que proyecte los más altos niveles de liderazgo, excelencia, } \\
\text { eficiencia y competitividad. }\end{array}$} \\
\hline \multicolumn{2}{|c|}{ III. DESCRIPCIÓN DE FUNCIONES PRINCIPALES } \\
\hline \multicolumn{2}{|c|}{$\begin{array}{l}\text { Planificar, elaborar y gestionar el presupuesto del departamento, bajo unos estándares } \\
\text { de recursos. } \\
\text { Analizar las acciones de la Unidad y evaluar y controlar los resultados de las mismas. } \\
\text { Diseñar y liderar la implementación del Plan de Marketing de la institución. } \\
\text { Asignar cargos, deberes y fijar objetivos para el personal de la Unidad. } \\
\text { Dirigir y liderar el equipo de trabajo. }\end{array}$} \\
\hline \multicolumn{2}{|c|}{ IV. COMPLEJIDAD DEL CARGO } \\
\hline \multicolumn{2}{|c|}{$\begin{array}{l}\text { La naturaleza del cargo implica un alto grado de responsabilidad en la toma de decisiones, compromiso, } \\
\text { planteamiento de objetivos, diseño y puesta en marcha de estrategias, fijación de metas y la verificación del alcance } \\
\text { de estas. }\end{array}$} \\
\hline \multicolumn{2}{|c|}{ V. REQUISITOS DE ESTUDIO Y EXPERIENCIA } \\
\hline Estudios & Experiencia \\
\hline $\begin{array}{l}\text { Contar con educación de nivel superior en } \\
\text { Ingeniería de mercados, Administración de } \\
\text { empresas, carreras afines o posgrados con } \\
\text { énfasis en mercadeo. }\end{array}$ & $\begin{array}{l}\text { Mínimo dos años de experiencia en organizaciones del mismo sector o } \\
\text { en labores afines al cargo. }\end{array}$ \\
\hline
\end{tabular}




\section{MANUAL ESPECIFICO DE FUNCIONES DE LA UNIDAD DE MERCADEO DE LA UNIVERSIDAD SIMÓN BOLÍVAR}

I. IDENTIFICACIÓN DEL CARGO

\begin{tabular}{|l|l}
\hline Cargo: & Director de Investigación de mercados \\
\hline Área: & Mercadeo \\
\hline II. OBJETIVOS
\end{tabular}

Obtención, el registro y el análisis de datos relativos a los ambientes, mercados y operaciones de mercadeo con objeto de proporcionar información útil para la identificación y solución de los diversos problemas de la institución, así como para la toma de decisiones adecuadas en el momento oportuno y preciso.

III. DESCRIPCIÓN DE FUNCIONES PRINCIPALES

Planea y define los Estudios de Investigación de Mercado

Evalúa y valida los resultados de los diversos estudios de mercado.

Supervisa la ejecución de los de los estudios y evalúa los resultados obtenidos.

Define y evalúa métodos y procedimientos para la recolección de información estadística, así como estudios cualitativos, cuantitativos y de segmentación.

IV. COMPLEJIDAD DEL CARGO

La naturaleza del cargo implica un alto grado de responsabilidad en la toma de decisiones, compromiso, planteamiento de objetivos, diseño y puesta en marcha de estrategias, fijación de metas y la verificación del alcance de estas. Trabajar en equipo con el Departamento de Admisiones

V. REQUISITOS DE ESTUDIO Y EXPERIENCIA

\begin{tabular}{|l|l|}
\hline Estudios & Experiencia \\
\hline $\begin{array}{l}\text { Contar con educación de nivel superior en Ingeniería de } \\
\text { mercados, Administración de empresas, carreras afines o }\end{array}$ & $\begin{array}{l}\text { Mínimo dos años de experiencia en organizaciones } \\
\text { del mismo sector o en labores afines al cargo. }\end{array}$
\end{tabular}

posgrados con énfasis en mercadeo.

\section{MANUAL ESPECIFICO DE FUNCIONES DE LA UNIDAD DE MERCADEO DE LA UNIVERSIDAD SIMÓN BOLÍVAR}

I. IDENTIFICACIÓN DEL CARGO

\begin{tabular}{|l|l}
\hline Cargo: & Director de CRM \\
\hline Área: & Mercadeo \\
\hline
\end{tabular}

\section{OBJETIVOS}

Conocer y manejar a la perfección programas de CRM, que compilará información que proviene de diversos canales: la página web de la institución, teléfono de contacto, chat, correo electrónico, material de marketing y redes sociales.

III. DESCRIPCIÓN DE FUNCIONES PRINCIPALES

Crear perfiles de usuario mediante diferentes estrategias.

Entender sus necesidades.

Fortalecer la relación con los usuarios mediante servicios adaptados y perfeccionados para cada perfil.

Optimizar la calidad de la relación con el cliente.

Automatizar actividades de fidelización del cliente.

IV. COMPLEJIDAD DEL CARGO

Sigue procedimientos preestablecidos en la ejecución de su labor y debe tener cuidado con el adecuado manejo de la información.

Manejar programas como SAP, ERP-CRM, cloud computing y SaaS.

Trabajar en equipo con el Departamento de Admisiones.

V. REQUISITOS DE ESTUDIO Y EXPERIENCIA

\begin{tabular}{|l|l|}
\hline Estudios & Experiencia \\
\hline $\begin{array}{l}\text { Profesional en administración, ingeniería de mercados, } \\
\text { comunicación y Periodismo, con posgrados y experiencia en } \\
\text { marketing. }\end{array}$ & $\begin{array}{l}\text { Mínimo 1 año de experiencia en organizaciones } \\
\text { del mismo sector o en labores afines al cargo. }\end{array}$ \\
\hline
\end{tabular}




\section{MANUAL ESPECIFICO DE FUNCIONES DE LA UNIDAD DE MERCADEO DE LA UNIVERSIDAD SIMÓN BOLÍVAR}

I. IDENTIFICACIÓN DEL CARGO

\begin{tabular}{|l|l|}
\hline Cargo: & Director de comunicaciones y relaciones públicas \\
\hline Área: & Mercadeo \\
\hline
\end{tabular}

II. OBJETIVOS

Planear, organizar, ejecutar y controlar las actividades de comunicaciones internas, externas y de publicidad que involucren todas las audiencias de la Universidad; y a la vez, direccionar los servicios creativos a nivel interno y externo, con el fin de crear una imagen e identidad institucional.

III. DESCRIPCIÓN DE FUNCIONES PRINCIPALES

Desarrollo plan de promoción y publicidad de programas (pregrados y posgrados)

Mediación con medios de comunicación masiva de la región

Mediación con empresas de publicidad exterior

Gestionar activaciones de marca institucional en eventos locales y nacionales.

Planear y dirigir acciones de servicios creativos.

Planear y dirigir estrategias de marketing digital.

Gestión de constante producción de Material POP.

Gestión de nuevos canales de distribución de contenido institucional.

IV. COMPLEJIDAD DEL CARGO

La naturaleza del cargo implica un alto grado de fluidez verbal, seguridad, confianza en sí mismo, percepción y análisis. Alta capacidad para toma de decisiones, liderazgo y creatividad.

V. REQUISITOS DE ESTUDIO Y EXPERIENCIA

Estudios

Profesional en comunicación social y periodismo, relaciones

Experiencia

Mínimo 1 año de experiencia en organizaciones

públicas, diseño gráfico y/o posgrados en mercadeo. del mismo sector o afines al cargo.

\section{MANUAL ESPECIFICO DE FUNCIONES DE LA UNIDAD DE MERCADEO DE LA UNIVERSIDAD SIMÓN BOLÍVAR}

I. IDENTIFICACIÓN DEL CARGO

\begin{tabular}{|l|l}
\hline Cargo: & Community manager \\
\hline Área: & Comunicaciones y relaciones públicas \\
\hline
\end{tabular}

II. OBJETIVOS

Construir, gestionar y administrar la comunidad online alrededor de la marca institucional en Internet, creando y manteniendo relaciones estables y duraderas con sus clientes, sus fans y, en general, cualquier usuario interesado en la marca.

III. DESCRIPCIÓN DE FUNCIONES PRINCIPALES

Coordinar y gestionar estrategias SEO y SEM

Administración de Social Media - Redes sociales.

Analítica Web - Análisis de tráfico en la página web, posicionamiento, visibilidad, entre otros.

Mobile Marketing.

Video Marketing.

Nuevas tendencias.

IV. COMPLEJIDAD DEL CARGO

La naturaleza del cargo implica buena capacidad comunicativa, empatía, moderación y alto conocimiento de la marca institucional.

Debe ir de la mano con la dirección de CRM e Investigación de mercados

V. REQUISITOS DE ESTUDIO Y EXPERIENCIA

\begin{tabular}{|l|l|}
\hline Estudios & Experiencia \\
\hline
\end{tabular}

\begin{tabular}{|l|l|}
\hline Profesional en Ingeniería de sistemas, de mercados, industrial. & Mínimo 1 año de experiencia en organizaciones del
\end{tabular}

Administración de empresas, comunicación social, y áreas afines. mismo sector o en labores afines al cargo.

Posgrados en community manager, marketing digital, etc. 


\begin{tabular}{|c|c|}
\hline $\begin{array}{r}\text { MANUAL ESPECIFICO DE FUNCIC } \\
\text { DE LA UNIVERSID }\end{array}$ & $\begin{array}{l}\text { DE LA UNIDAD DE MERCADEO } \\
\text { MÓN BOLÍVAR }\end{array}$ \\
\hline I. IDENTIFICACIÓN DEL CARGO & \\
\hline Cargo: & Servicios creativos \\
\hline Área: & Comunicaciones y relaciones públicas \\
\hline II. OBJETIVOS & \\
\hline $\begin{array}{l}\text { Construir, gestionar y controlar presentaciones de camm } \\
\text { concreta el objetivo estratégico de la marca, definiendo } \\
\text { campaña, a que personas se dirige y qué mensaje se les } \\
\text { se desarrollan y ponen en marcha las grandes ideas. }\end{array}$ & $\begin{array}{l}\text { segurarse de la calidad de la misma. En él se } \\
\text { amente lo que se pretende conseguir con una } \\
\text { mitir. Es el núcleo imaginativo de la Unidad, donde }\end{array}$ \\
\hline III. DESCRIPCIÓN DE FUNCIONES PRINCIPALES & \\
\hline $\begin{array}{l}\text { Diseño de artes y piezas publicitarias de manera oportu } \\
\text { Registro fotográfico. } \\
\text { Diseño de material POP } \\
\text { Musicalización (fondos musicales y anuncios cantados) } \\
\text { Materiales audiovisuales } \\
\text { Producción de anuncios para cada medio } \\
\text { Dar forma a los mensajes a transmitir. }\end{array}$ & \\
\hline IV. COMPLEJIDAD DEL CARGO & \\
\hline $\begin{array}{l}\text { Mentalidad creativa, talento artístico, capacidad para } \\
\text { excelente nivel cultural y estar actualizado constantem }\end{array}$ & $\begin{array}{l}\text { jo presión y en equipo, sentido del humor, } \\
\text { aaja para todas las áreas de la organización. }\end{array}$ \\
\hline Estudios & Experiencia \\
\hline $\begin{array}{l}\text { Profesional en Diseño gráfico, ingeniería de mercados, } \\
\text { multimedia, de sistemas, publicidad, y áreas afines. }\end{array}$ & $\begin{array}{l}\text { Mínimo } 1 \text { año de experiencia en organizaciones } \\
\text { del mismo sector o en labores afines al cargo. }\end{array}$ \\
\hline
\end{tabular}

\section{Conclusiones y Recomendaciones}

El marketing es indispensable en todas las organizaciones al realizarse y gestionarse de forma organizada, coordinada y estructurada, para que los esfuerzos sean exitosos y se vean reflejados en la adquisición del servicio ofrecido, la fidelización de los usuarios (en este caso estudiantes) a la institución, reconocimiento en el mercado, y además, conseguir un posicionamiento positivo en la mente de la comunidad interna y externa.

A partir de los resultados presentados se concluye como las estrategias de marketing tradicional y las estrategias de promoción en medios digitales realizadas por la institución han arrojado resultados positivos en el trascurso del tiempo, sin embargo, dadas las exigencias actuales del entrono en general y el mercado educativo, deben planearse y ejecutarse acciones y estrategias más eficientes y eficaces, con una mayor coordinación; para lo cual es de mucha utilidad formar una unidad debidamente estructurada encargada de direccionar el proceso de mercadeo, y así, optimizar los esfuerzos de su personal y contribuir al crecimiento y mejoramiento de los procesos, servicios, imagen de la institución, servicio al cliente, difusión y gestión de su oferta académica. Si bien, los esfuerzos realizados por la institución hasta el momento han sido muy bien ejecutados y acertados, es indispensable ir evolucionando y adaptando las acciones de marketing a las tendencias y exigencias del mercado, además de permitir compactar las estrategias realizadas hacia un mismo foco, generando mayor sinergia en las acciones y por supuesto, resultados óptimos y con un mayor alcance dentro del mercado. 
Por tales motivos es indispensable y recomendable para las Instituciones de Educación Superior, que estan en proceso de crecimiento y mejoramiento, como lo es la estudiada en el presente proyecto; evaluar la posibilidad de desarrollar e implementar una Unidad de Mercadeo, con su debido direccionamiento, estructurada organizacionalmente, con manuales de funciones actualizados $y$ tareas específicas a cada cargo que se ejecuta acorde a sus necesidades y características organizacionales.

De este modo, se recomienda para la aplicación y desarrollo de propuestas similares, sea fundamental identificar los factores clave en el desempeño actual y las exigencias del mercado al cual se enfrenta la institución y a partir de estos, revisar las funciones generales y específicas de la Unidad de Mercadeo tomando como base las aquí expuestas, y por supuesto, establecer una estructura acorde a sus necesidades y capacidades. Además es indispensable tener en cuenta la articulación con las diferentes dependencias de la universidad permitiendo generar un trabajo articulado desde la dirección hacia todas las áreas requeridas dentro de la IES.

\section{REFERENCIAS}

AMA. (1960). Marketing Definitions: A Glossary of Marketing Terms.Chicago: AMA.

Álvarez, M. (1996). Manual para Elaborar Manuales de Políticas y procedimientos. México, D.F.: Panorama.

Bernal, J. (2006). Metodología de la investigación para administración, economía humanidades y ciencias sociales. México D.F.: Pearson Education.

Chumaceiro, A. Hernández, J. y Chirinos, E. (2016). Responsabilidad social universitaria, desarrollo sostenible y ciudadanía ambiental. Cuadernos de RSO. 4(1). 53-64.
Denzin, N. \& Lincon, Y. (Eds.) (1994). Handbook of Qualitative Research. Londren: Sage Publications.

Díaz, M. (2009). Metodología de la investigación científica y bioestadística. Santiago de Chile: Ril.

Jiménez, C. (2012). Análisis de la competencia. Caracas: IESA.

Kotler, P. y Armstrong, G. (2008). Fundamentos de marketing (8 ed.). México, D.F.: Pearson Educación.

Farías, P. (2014). Estrategias de marketing utilizadas por las empresas chilenas para incrementar el valor de los clientes. Cuadernos de Administración, 30(51). 8-14. Disponible en http://www.scielo.org.co/pdf/ cuadm/v30n51/v30n51a02.pdf

Hernández, J. y Chumaceiro, A. (2008). Sociedad del conocimiento y pertinencia social universitaria. CEISEP una propuesta para la UNERMB. Revista Venezolana de Ciencias Sociales. 12(1). 77-92.

Hernández, J., Chumaceiro, A. y Reyes, I. (2016). Estado y pertinencia social universitaria en Venezuela. Aproximación al desarrollo endógeno. En G. Ziritt, J. Hernández, J. Barboza y M. Padrón (Comp.), Estado, universidad y sociedad. Mirando la educación universitaria desde el desarrollo endógeno. (66-86). Cabimas: Fondo editorial Universidad Nacional Experimental-UNERMB. Recuperado de http://150.185.9.18/ fondo_editorial/images/PDF/ProgramaInvestigacion/Estado $\% 20$ Universidad $\% 20 y \% 20$ Sociedad $\% 20$ VD.pdf

Llorente, J. (2017). Marketing educativo: Captación y fidelización de alumnos. Madrid: ESIC.

Martinez, A., Ruiz, C. y Escrivá, J. (2014). Marketing en la actividad comercial. Madrid: McGraw Hill. 
Malhotra, N. (2004). Investigación de mercados, un enfoque aplicado. México D.F.: Pearson Education.

Ponce, H. (2006). La matriz FODA: una alternativa para realizar diagnósticos y determinar estrategias de intervención en las organizaciones productivas y sociales. Contribuciones a la Economía, (5). Recuperado de http://www.eumed. net/ce/2006/hpt-FODA.htm

Rojas, D. (2013). La biblia del marketing. Barcelona: Lexus.

Rodríguez, J. (2012). Cómo elaborar y usar los manuales administrativos. México, D.F.: Cengage Learning .

Schnarch, D. (2013). Marketing para Pymes: un enfoque para Latinoamerica. México, D.F.: Alfaomega.

\section{Biodata}

Eimys Vanessa Romero Corro es Ingeniera de Mercados con diplomado en gestión de las tecnologías de la información de la Universidad Simón Bolívar (Colombia). Perteneciente al semillero de investigación de la Universidad Simón Bolívar (Colombia). Estudiante de intercambio en la escuela de Ingeniería Comercial en el primer periodo del 2016, facultad de Ciencias económicas y administrativas de la Universidad de Valparaíso (Chile). Con distinciones a la Excelencia académica en la Universidad Simón Bolívar durante los semestres 2016-2 y 2017-2. https:/orcid. org/0000-0003-2683-3249

Uliana María Contreras Buelvas es Ingeniera de Mercados con diplomado en gestión de las tecnologías de la información en la Universidad Simón Bolívar (Colombia). Perteneciente al semillero de investigación de la Universidad Simón Bolívar, (Colombia). Estudiante de intercambio en la escuela de Ingeniería Comercial en el primer periodo del 2016, facultad de Ciencias económicas y administrativas de la Universidad de Valparaíso (Chile). https:// orcid.org/0000-0001-9307-4591 